\title{
Laboratórios Remotos Aumentados para o ensino de Circuitos Elétricos
}

\author{
Priscila Cadorin Nicolete ${ }^{1}$, Eduardo Oliveira Junior ${ }^{2}$, Liane Margarida \\ Rockenbach Tarouco ${ }^{2}$, Eduardo de Vila ${ }^{3}$, Marta Cristiano ${ }^{3}$, Aline Coelho dos \\ Santos, Juarez Bento Silva ${ }^{3}$
}

${ }^{1}$ Pós-Graduação em Informática na Educação - Universidade Federal do Rio Grande do Sul (UFRGS). Av Paulo Gama 110, Anexo III, 344 - Porto Alegre (RS) Brasil

${ }^{2}$ Instituto Federal de Santa Catarina (IFSC). Av. XV de Novembro, 61 - Araranguá (SC) Brasil

${ }^{3}$ Laboratório de Experimentação Remota - Universidade Federal de Santa Catarina (UFSC). Rua Pedro João Pereira150, Mato Alto - Araranguá (SC) Brasil

priscilanicolete@hotmail.com, eduardo.junioreifsc.edu.br, lianedpenta.ufrgs.br

\begin{abstract}
The union of Augmented Reality techniques with Remote Laboratories shapes augmented Remote Laboratories (ARL), allowing users of these laboratories to gain deeper experiences in their educational practices. In this sense, the study presented here consists of the presentation of an LRA in order to create new opportunities for the teaching of the basic concepts of electricity and electric circuits.
\end{abstract}

Resumo. Os Laboratórios Remotos Aumentados (LRA) configuram-se pela união de técnicas de Realidade Aumentada com Laboratórios Remotos, permitindo que os usuários desses laboratórios obtenham experiências mais profundas em suas práticas educacionais. Nesse sentido, o estudo aqui exposto consiste na apresentação de um LRA a fim de criar novas oportunidades para o ensino dos conceitos básicos de eletricidade e circuitos elétrico.

\section{Introdução}

Os Laboratórios Remotos e Virtuais têm se mostrado eficientes na superação de certas limitações dos laboratórios tradicionais de ensino prático, tais como altos custos, disponibilidade limitada e preço elevado de manutenção.

Os Laboratórios Virtuais (LV) são softwares que possibilitam que estudantes manipulem fenômenos, que são representações computacionais da realidade. Os Laboratórios Remotos (LR), por sua vez, são experimentos reais que podem ser acessados a distância, esse acesso é realizado por meio de alguma interface que realiza a mediação entre o aluno e o equipamento (Rodriguez-Gil et al., 2017).

Estes laboratórios são ótimas opções para instituições de ensino que não possuem laboratórios presenciais, ou que pretendem estender seus recursos escassos, ou ainda que desejem compartilhar equipamentos com outras instituições, e dessa forma permitir que um maior número de alunos obtenha conhecimentos práticos em diversas áreas do conhecimento. Além disso, o uso desses laboratórios não está limitado a uma sala de laboratório, em um horário específico, como nos laboratórios hands-on, essas tecnologias permanecem disponíveis aos estudantes em todo o tempo, permitindo o acesso sete dias por semana, 24 horas por dia, podendo ser explorado principalmente no ensino à distância (Antonio, Simão, Alves, Silva, \& Santos, 2016; Zubía et al., 2017). 
VIII Congresso Brasileiro de Informática na Educação (CBIE 2019)

Anais do XXV Workshop de Informática na Esçla (WIE 2019)

Estudos recentes têm mostrado a importância em unir a potencialidade de ambas tecnologias - Laboratórios Virtuais e Remotos - a fim de superar limitações inerentes a esses laboratórios e enriquecer as práticas pedagógicas. Nesse contexto, surge os Laboratórios Remotos Aumentados (LRA), ou Laboratórios Remotos de Realidade Aumentada, que pretendem misturar elementos virtuais e remotos em um único laboratório, por meio de técnicas de Realidade Aumentada (Mejías \& Andújar, 2012).

A Realidade Aumentada (RA) é uma tecnologia que tem como objetivo complementar o mundo real com objetos gerados por computador, fazendo com que elementos virtuais e reais coexistem no mesmo espaço no mundo real (Azuma et al., 2001). Essa sobreposição amplia o acesso a informação gerando novas oportunidades de interação (Frank \& Kapila, 2017). Diante disso, os Laboratórios Remotos Aumentados pretendem contribuir na superação de certas limitações impostas pelos Laboratórios Remotos, tais como o feedback em fluxo de vídeo, que na maioria das vezes é uma visualização simplificada, que não permite a total exploração da experiência.

Conforme Andújar e Márquez (2012), os Laboratórios Remotos que incorporam RA é um passo adiante nos processos de ensino e aprendizagem apoiados em laboratório online, uma vez que é capaz de conectar o mundo real do laboratório remoto e o mundo virtual dos laboratórios virtuais para configurar cenários dos quais a realidade e a virtualidade interagem umas com as outras, moldando nossos ambientes educacionais.

Neste contexto, este estudo visa apresentar o desenvolvimento de um Laboratório Remoto Aumentado, a partir de um LR já existente, a fim de disponibilizar aplicações sofisticadas aos estudantes que poderá resultar em uma aprendizagem mais profunda. $\mathrm{O}$ aprimoramento de um laboratório remoto já existente visa entender de que forma é possível reaproveitar recursos para criar novos arranjos de experimentação prática.

\section{Materiais e Métodos}

Para efetivação dessa pesquisa, foi necessário a conclusão das seguintes etapas: (i) mapear as etapas de construção de um laboratório remoto aumentado, por meio de uma pesquisa bibliográfica; (ii) determinar, junto ao professor da disciplina, qual laboratório remoto seria aumentado e quais aspectos seriam aumentados no experimento, e por fim o (iii) desenvolvimento do Laboratório Remoto Aumentado.

\section{O Painel Elétrico AC Aumentado}

O LRA foi desenvolvido a partir de um laboratório remoto já existente, disponibilizado pelo Laboratório de Experimentação Remota - Rexlab, o "Painel Elétrico AC". Este experimento aborda as associações em série, paralela e mista em redes de corrente alternada. Nele é possível observar a intensidade luminosa de seis lâmpadas variar de acordo com a configuração do circuito, para isso quatro chaves são dispostas em diferentes pontos e controladas pelo usuário.

A RA foi utilizada neste experimento para demonstrar de forma lúdica como a corrente elétrica percorre o circuito, mostrando a presença, ausência e sua intensidade por meio de animação e composição de cores. Além disso, também foram acrescentados os valores das correntes em cada ponto do circuito. Como marcador para a RA, é utilizado o próprio streaming de vídeo do experimento remoto. Assim, o estudante irá apontar seu smartphone para a tela do computador para visualizar e interagir com laboratório remoto aumentado. A figura 1 apresenta como ocorre acesso ao LRA "Painel Elétrico AC Aumentado". 


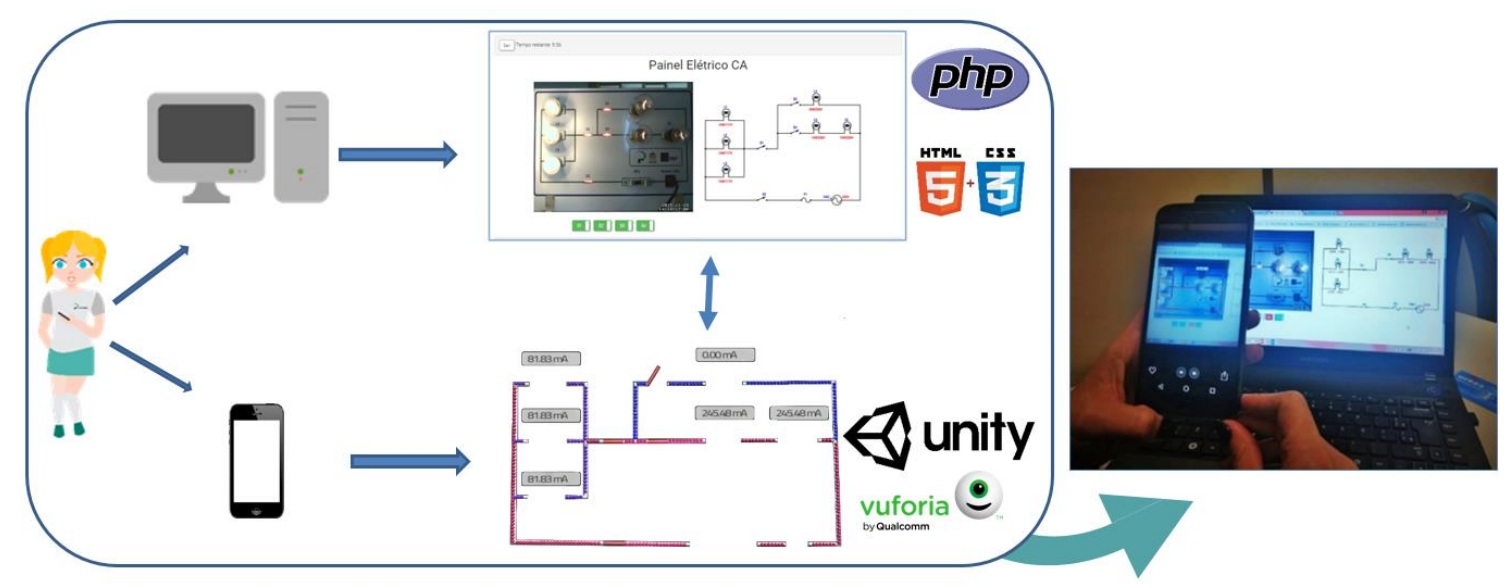

Figura 1 - Funcionamento do acesso ao LRA "Painel Elétrico AC Aumentado".

\subsection{Arquitetura dos Laboratórios Remotos disponibilizados pelo Rexlab}

Todos os laboratórios remotos desenvolvidos pelo Rexlab são implementados a partir da arquitetura padronizada, de hardware e software básico. A diferenciação entre os experimentos está apenas nos tipos diferentes de sensores e atuadores, que são instalados de acordo com as especificidades dos experimentos remotos disponibilizados.

A arquitetura é dividida em quatro módulos: (i) Laboratório Real que trata dos componentes reais - sensores e atuadores integrados em uma placa de controle e aquisição; (ii) Computador Embarcado que compreende a parte de disponibilização do experimento na rede, que consiste em um serviço Node.js hospedado em um Single-Board Computer (SBC), normalmente um Raspberry pi, que também gerencia e disponibiliza o serviço de streaming gerado por uma câmera web com conexão USB; (iii) RELLE (Remote Labs Learning Environment), que é o sistema de gerenciamento dos experimentos; e (iv) Cliente que trata do modelo de acesso utilizado para manipulação dos experimentos (Lima, Simão, Silva, \& Rochadel, 2015a).

O cliente web, disponibilizado pelo sistema RELLE, é desenvolvido em PHP 5.5, sendo seu frontend desenvolvido em HTML, fazendo uso do framework CSS Bootstrap, em conjunto à biblioteca JavaScrip jQuery. O RELLE também garante que dois usuários não acessem o experimento ao mesmo tempo. A partir do botão "Acessar" o sistema dispara um evento para comunicação com a Web API FCFS e desse modo, se houver outro usuário utilizando o experimento, o usuário será inserido em uma fila respeitando a ordem de chegada (fila FIFO - First In, First Out). Caso contrário, o sistema disponibiliza um token de sessão e o cliente obtém a permissão para acessar e carregar todos arquivos (html, css e js). Após carregar o cliente para o Smart Device (client.js), uma conexão WebSocket com este dispositivo é estabelecida (Lima, Simão, Silva, \& Rochadel, 2015b).

\subsection{Arquitetura desenvolvida para o Laboratório Remoto Aumentado}

Os objetos 3D desenvolvidos para a composição do Laboratório Remoto Aumentado foram modelados por meio da ferramenta Unity 3D (2018), um motor de jogo que permite o desenvolvimento de aplicativos com uso de imagens $3 \mathrm{D}$, que nativamente oferece suporte ao desenvolvimento para Android. A linguagem de programação empregada foi C\#, uma das linguagens padrão de desenvolvimento em Unity 3D. 
VIII Congresso Brasileiro de Informática na Educação (CBIE 2019)

Anais do XXV Workshop de Informática na Escqla (WIE 2019)

Para a integração das tecnologias de RA, utilizou-se o framework Vuforia, que está disponível gratuitamente e oferece uma plataforma de visão computacional que permite a construção de experiências interativas e que apresenta diversos recursos relacionados à realidade aumentada (Herpich, Guarese, \& Tarouco, 2017). Através de uma série de abstrações, o Vuforia possibilita que se relacionem marcadores, isto é, imagens de alto contraste presentes em um banco de dados, com modelos tridimensionais programáveis, desenvolvidos em Unity. Assim, quando o algoritmo da própria API detecta através da câmera a presença de um marcador conhecido, gera na tela o modelo tridimensional correspondente.

Para que a RA pudesse interagir com hardware do laboratório remoto de forma bidirecional foi necessário o desenvolvimento de um protocolo de comunicação entre o aplicativo de RA e o LR, de modo que toda a alteração realizada nos objetos virtuais refletisse no laboratório real, da mesma forma que as alterações no LR refletisse na RA.

Desse modo, essa comunicação foi implementada através de um sistema de web sockets, no qual, a cada interação do usuário, pacotes de informação são enviados do app de RA cliente diretamente ao servidor, que os processa e aplica as mudanças no experimento real de acordo com os dados recebidos. Para implementar o sistema este sistema, foi utilizada SocketIO, uma biblioteca Javascript/C\# que oferece funcionalidades para comunicação em tempo real entre clientes e servidores através da web.

Detalhadamente, a comunicação entre o aplicativo desenvolvido em Unity e o laboratório remoto é feita em três etapas, a primeira etapa consiste no armazenamento do token no Raspberry (Computador Embarcado). Após o usuário possuir a permissão para acessar o experimento, o Raspberry armazena o token da sessão em uma variável. Esse token servirá para conceder permissão de acesso a aplicação de RA para acessar a sessão que foi aberta pela página web do experimento, uma vez que o RELLE bloqueia o acesso de mais de um cliente ao experimento ao mesmo tempo. Depois, ao acessar a aplicação de Realidade Aumentada, um script utilizando a biblioteca SocketIO no C\# envia uma solicitação de conexão para o Raspberry. Neste momento é estabelecido a conexão entre a RA e o Laboratório Remoto.

Com a conexão estabelecida, inicia-se as trocas de informações entre a aplicação de RA, Raspberry e a página do experimento. Quando a RA envia uma informação para alterar o estado do experimento, o Raspberry utiliza a variável que está armazenado o token da sessão e envia essas informações (chaves para alterar enviadas pela RA e o token) para a página do experimento, a página altera os estados das chaves presentes na página e envia novamente para o Raspberry, o qual enviará os comandos para a placa de aquisição e controle para a alteração do estado do laboratório real. Do mesmo modo, se o usuário alterar os estados das chaves do experimento na página web, isso refletirá na RA. A página envia as alterações para o Raspberry e o Raspberry envia para a RA.

O usuário tem acesso a RA por meio do aplicativo avatAR-LRA, que pode ser encontrado na loja de aplicativo da Google - Play Store.

\section{Considerações Finais}

O estudo teve como objetivo conhecer de que forma o uso de Realidade Aumentada em Laboratórios Remotos pode contribuir para o aprimoramento das práticas pedagógicas. Para isso, foi desenvolvido um Laboratório Remoto Aumentado, a partir de um laboratório remoto já existente, para apoiar o ensino de circuitos elétricos. 
Com isso, percebeu-se que a RA para esses laboratórios permite apresentar detalhes do experimento que antes seria impossível por meio das técnicas tradicionais dos laboratórios remotos. Por meio da RA, objetos reais podem ser combinados com elementos virtuais fazendo com que novas informações sejam levadas para os alunos, contribuindo para uma maior compreensão dos conceitos em estudo.

Além disso, essa união pode criar novos oportunidades de aprendizagem prática a partir de um mesmo experimento, ou seja, conforme a composição dos elementos virtuais, um único laboratório remoto pode ser utilizado para diferentes objetivos educacionais. Por exemplo, o Laboratório Remoto Aumentado apresentado neste estudo será aplicado na primeira parte da disciplina de Medidas Eletromecânica de um curso Técnico em Eletromecânica com objetivo de apoiar o ensino dos conceitos básicos de eletricidade, tais como, Lei de OHM, divisão de corrente e divisão de tensão. Porém, em uma segunda versão serão incluídos elementos de medição virtuais que apresentarão medições reais do experimento e essa nova versão será utilizada para o ensino e manipulação dos instrumentos básicos de medidas elétricas, podemos citar o voltímetro e o amperímetro.

Portanto, os Laboratórios Remotos Aumentados, além de contribuir na superação das limitações dos laboratórios remotos aprimorando o feedback apresentado aos estudantes, surgem como uma ótima opção para o reaproveitamento de recursos, uma vez que um mesmo experimento remoto pode criar diferentes arranjos de experimentação prática.

\section{Referências}

Antonio, C. P., Simão, J. P. S., Alves, J. B. d. M., Silva, J. B. d., \& Santos, A. C. d. S. (2016). Using 3D Virtual Worlds Integrated to Remote Experimentation in Sciences Teaching. Gamification-Based E-Learning Strategies for Computer Programming Education, 195.

Azuma, R., Baillot, Y., Behringer, R., Feiner, S., Julier, S., \& MacIntyre, B. (2001). Recent advances in augmented reality: NAVAL RESEARCH LAB WASHINGTON DC.

Frank, J. A., \& Kapila, V. (2017). Mixed-reality learning environments: Integrating mobile interfaces with laboratory test-beds. Computers \& Education, 110, 88-104.

Herpich, F., Guarese, R. L. M., \& Tarouco, L. M. R. (2017). A comparative analysis of augmented reality frameworks aimed at the development of educational applications. Creative Education, 8(09), 1433.

Lima, J. P. C., Simão, J. P. S., Silva, J. B., \& Rochadel, W. (2015a). GT-MRE: Grupo de Trabalho em Experimentação Remota Móvel - RF - Relatório Final. Araranguá: Relatório final - Fase 1.

Lima, J. P. C., Simão, J. P. S., Silva, J. B., \& Rochadel, W. (2015b). GT-MRE: Grupo de Trabalho em Experimentação Remota Móvel - RT2 - Avaliação dos resultados do protótipo. Araranguá: Relatório Técnico 2 - Fase 1.

Mejías, A. B., \& Andújar, J. M. (2012). A Pilot Study of the Effectiveness of Augmented Reality to Enhance the Use of Remote Labs in Electrical Engineering Education. [Article]. Journal of science education and technology, 21(5), 540-557.

Rodriguez-Gil, L., Zubia, J. G., Orduña, P., \& Lopez-de-Ipina, D. (2017). Towards new multiplatform hybrid online laboratory models. IEEE Transactions on Learning Technologies, 10(3), 318-330.

Zubía, J., G, Cuadros, J., Romero, S., Hernandez-Jayo, U., Orduña, P., Guenaga, M., Gustavsson, L. (2017). Empirical Analysis of the Use of the VISIR Remote Lab in Teaching Analog Electronics. IEEE Transactions on Education, 60(2), 149-156. 